\title{
Classical and quantum plasmonics in graphene nanodisks Role of edge states
}

Christensen, Thomas; Wang, Weihua; Jauho, Antti-Pekka; Wubs, Martijn; Mortensen, N. Asger

\section{Published in:}

Physical Review B

Link to article, DOI:

10.1103/PhysRevB.90.241414

Publication date:

2014

Document Version

Publisher's PDF, also known as Version of record

Link back to DTU Orbit

Citation (APA):

Christensen, T., Wang, W., Jauho, A-P., Wubs, M., \& Mortensen, N. A. (2014). Classical and quantum plasmonics in graphene nanodisks: Role of edge states. Physical Review B, 90(24), 241414.

https://doi.org/10.1103/PhysRevB.90.241414

\section{General rights}

Copyright and moral rights for the publications made accessible in the public portal are retained by the authors and/or other copyright owners and it is a condition of accessing publications that users recognise and abide by the legal requirements associated with these rights.

- Users may download and print one copy of any publication from the public portal for the purpose of private study or research.

- You may not further distribute the material or use it for any profit-making activity or commercial gain

- You may freely distribute the URL identifying the publication in the public portal 


\title{
Classical and quantum plasmonics in graphene nanodisks: Role of edge states
}

\author{
Thomas Christensen, ${ }^{1,2}$ Weihua Wang, ${ }^{1,2}$ Antti-Pekka Jauho, ${ }^{2,3}$ Martijn Wubs, ${ }^{1,2}$ and N. Asger Mortensen ${ }^{1,2, *}$ \\ ${ }^{1}$ Department of Photonics Engineering, Technical University of Denmark, DK-2800 Kgs. Lyngby, Denmark \\ ${ }^{2}$ Center for Nanostructured Graphene, Technical University of Denmark, DK-2800 Kgs. Lyngby, Denmark \\ ${ }^{3}$ Department of Micro- and Nanotechnology, Technical University of Denmark, DK-2800 Kgs. Lyngby, Denmark
}

(Received 15 July 2014; revised manuscript received 4 November 2014; published 16 December 2014)

\begin{abstract}
Edge states are ubiquitous for many condensed matter systems with multicomponent wave functions. For example, edge states play a crucial role in transport in zigzag graphene nanoribbons. Here, we report microscopic calculations of quantum plasmonics in doped graphene nanodisks with zigzag edges. We express the nanodisk conductivity $\sigma(\omega)$ as a sum of the conventional bulk conductivity $\sigma_{\mathrm{B}}(\omega)$, and a novel term $\sigma_{\mathrm{E}}(\omega)$, corresponding to a coupling between the edge and bulk states. We show that the edge states give rise to a redshift and broadening of the plasmon resonance, and that they often significantly impact the absorption efficiency. We further develop simplified models, incorporating nonlocal response within a hydrodynamical approach, which allow a semiquantitative description of plasmonics in the ultrasmall size regime. Furthermore, we show that the effect of hydrodynamic and edge-conductivity corrections scale identically, approximately with the inverse of the disk radius, highlighting their equatable importance. However, the polarization dependence is only given by fully microscopic models. The approach developed here should have many applications in other systems supporting edge states.
\end{abstract}

DOI: 10.1103/PhysRevB.90.241414

PACS number(s): 73.22.Pr, 73.20.Mf, 78.20.Ci, 78.67.Wj

Introduction. Plasmonics at the nanoscale introduces a host of novel phenomena, both in terms of improved efficacy of certain classical phenomena, e.g., extreme field enhancements, but also conceptually by offering a tunable transition from the classical to the quantum regime [1]. Probing and understanding this transition in detail, and in particular the breakdown of classical predictions, is an important task in view of the progress in nanofabrication [2,3]. With the emergence of lowdimensional materials such as graphene, new avenues develop, both experimentally and theoretically. Graphene, and several other low-dimensional systems, exhibits an approximately linear, gapless, two-band energy dispersion $\epsilon= \pm \hbar v_{\mathrm{F}} k$, with Fermi velocity $v_{\mathrm{F}}$. The plasmonic consequences of this nonstandard dispersion and dimensionality have been investigated vigorously in recent years [4-10].

The accurate description of low-energy excitations in graphene by simple tight-binding (TB) Hamiltonians allows investigations of nonclassical plasmonic features of relatively large graphene structures $[11,12]$. It has recently been theoretically demonstrated that the optical excitations of fewatom graphene nanostructures involve multiple individual electron-hole pairs (EHPs) strongly modified by the Coulomb interaction, occasionally referred to as molecular plasmons [13]. Conversely, experimental measurements on ensembles of larger disks, of radii $R \gtrsim 50 \mathrm{~nm}$, have exhibited distinctly classical features $[14,15]$. Improving our understanding of this transition between opposing realms is underscored by the many advances in fabrication of graphene nanostructures [16-18].

In this Rapid Communication we show that for smaller graphene disks, though larger than $R \gtrsim 7 \mathrm{~nm}$, two essential modifications of the classical single-disk response arise, due to edge states and to nonlocal response, producing an overall

\footnotetext{
*asger@mailaps.com
}

redshift and broadening of the dipole resonance. In particular, we show that the existence of edge states due to zigzag (ZZ) features can be accounted for via an edge-state conductivity, while the impact of nonlocal response can be accounted for effectively within a hydrodynamic model. This affirms and extends the supposition regarding the crucial role of edge states in prior numerical work [11]. In Fig. 1 we outline and summarize the different computational approaches considered in this Rapid Communication.

Electronic states. The simplest atomistic description of the conduction electrons of graphene, without explicit treatment of spin, is given by the $p_{z}$-orbital nearest-neighbor TB Hamiltonian with hopping energy $t_{A B}=2.8 \mathrm{eV}$ :

$$
\hat{\mathcal{H}}_{\mathrm{TB}}=-t_{A B} \sum_{\left\langle j, j^{\prime}\right\rangle} \hat{a}_{j}^{\dagger} \hat{b}_{j^{\prime}}+\hat{b}_{j^{\prime}}^{\dagger} \hat{a}_{j}
$$

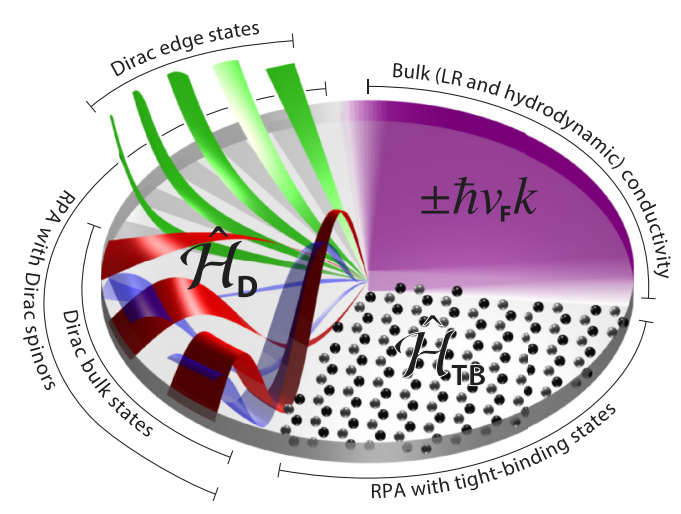

FIG. 1. (Color online) Illustration of considered levels of approximation for a graphene nanodisk. Angular slices of Dirac ZZ bulk state spinor components are indicated in red and blue, and edge state nonzero components in green. 
with $A$ - and $B$-sublattice annihilation (creation) operators $\hat{a}_{j}^{(\dagger)}$ and $\hat{b}_{j}^{(\dagger)}$ at sites $j$, and with $\left\langle j, j^{\prime}\right\rangle$ indicating summation over nearest neighbors. In the low-energy limit, for extended graphene, the characteristics of the TB approach are asymptotically reproduced by the four-spinor Dirac equation, $\hat{\mathcal{H}}_{\mathrm{D}} \boldsymbol{\psi}(\mathbf{r})=\epsilon \boldsymbol{\psi}(\mathbf{r})$, with the Hamiltonian [19]

$$
\hat{\mathcal{H}}_{\mathrm{D}}=v_{\mathrm{F}}\left(\tau_{0} \otimes \sigma_{x} \hat{p}_{x}+\tau_{z} \otimes \sigma_{y} \hat{p}_{y}\right),
$$

where $\hat{\boldsymbol{p}}=-i \hbar \nabla$ denotes momentum, and with Pauli matrices $\tau_{i}$ and $\sigma_{i}$ belonging to valley and sublattice subspaces, respectively. In the absence of valley mixing, the four-spinor equation for $\psi(\mathbf{r})=\left[\psi_{A}^{+}(\mathbf{r}), \psi_{B}^{+}(\mathbf{r}), \psi_{A}^{-}(\mathbf{r}), \psi_{B}^{-}(\mathbf{r})\right]^{\mathrm{T}}$ decouples into a pair of two-spinor equations for $\boldsymbol{\psi}^{\kappa}(\mathbf{r})=\left[\psi_{A}^{\kappa}(\mathbf{r}), \psi_{B}^{\kappa}(\mathbf{r})\right]^{\mathrm{T}}$ associated with valley indices $\kappa= \pm 1$ pertaining to Dirac valleys $\boldsymbol{K}^{\kappa}=[\sqrt{3}, \kappa]^{\mathrm{T}} 2 \pi / 3 a_{\mathrm{LC}}$, with lattice constant $a_{\mathrm{LC}}=$ $2.46 \AA$ А [20].

Finite graphene structures are easily modeled with Eq. (1) by omitting the absent neighbors in the matrix representation of $\hat{\mathcal{H}}_{\mathrm{TB}}$, whose dimension equals the number of constituent carbon atoms. For the continuum Dirac equation, Eq. (2), suitable boundary conditions (BCs) are needed. General considerations, enforcing no-spill current conditions, Hermiticity, and unitarity, lead to a rather broad family of allowable BCs [22,23], which can be made explicit by using the atomistic details of the structural termination. In the present work we consider $\mathrm{ZZ}$ lattice termination (which can be considered appropriate, in general, for nonarmchair minimal lattice terminations as argued in Ref. [23]) forcing a single sublattice component to vanish, e.g., forcing $\psi_{A}^{\kappa}(\mathbf{r})=0$ on the boundary if the $\mathrm{ZZ}$ edge belongs to the $B$-sublattice. For comparison we also consider the infinite mass (IM) BC [24], corresponding microscopically to confinement due to an atomically staggered potential [23], which enforces an intersublattice phase relationship $\psi_{B}^{\kappa}(\mathbf{r}) / \psi_{A}^{\kappa}(\mathbf{r})=i e^{i \kappa \theta}$, with $\theta$ denoting the tangential boundary angle [25].

Upon application of $\mathrm{BCs}$, the otherwise linear Dirac dispersion $\epsilon= \pm \hbar v_{\mathrm{F}} k$ is transformed into a discrete set of energies and associated spinors. For the case of a homogeneous disk of radius $R$, the nonzero-energy spinors are quantized in angular and radial quantum numbers $l=0, \pm 1, \pm 2, \ldots$ and $n=1,2, \ldots[26,27]$ :

$$
\psi_{l n}^{\kappa}(\tilde{r}, \theta)=\frac{e^{i l \theta}}{\sqrt{N_{l n}^{\kappa}}}\left[\begin{array}{c}
J_{l}\left(\beta_{l n} \tilde{r}\right) \\
i \kappa J_{l+\kappa}\left(\beta_{l n} \tilde{r}\right) e^{i \kappa \theta}
\end{array}\right],
$$

with normalization $N_{\ln }^{\kappa}$ [see Supplemental Material (SM) [28]] expressed through the dimensionless radial coordinate $\tilde{r}=r / R$ and momenta $\beta_{l n}=\epsilon_{l n} / \hbar \omega_{R}$ with circumferential fermion frequency $\omega_{R}=v_{\mathrm{F}} / R$. The $\mathrm{ZZ} \mathrm{BC} \mathrm{energies} \mathrm{are}$ valley independent and correspond to zeros of the Bessel function, i.e., $\beta_{l n}$ fulfills $J_{l}\left(\beta_{l n}\right)=0$, while the IM BC yields valley-dependent energies, given by $\kappa J_{l+\kappa}\left(\beta_{l n}^{\kappa}\right)=J_{l}\left(\beta_{l n}^{\kappa}\right)$. Additionally, for the ZZ BC a set of zero-energy spinors exist, here denoted by $\boldsymbol{\phi}_{\ell}^{\kappa}$, discretized in angular quantum numbers $\ell=0,1, \ldots, \ell_{\max }[26,27]$ :

$$
\boldsymbol{\phi}_{\ell}^{\kappa}(\tilde{r}, \theta)=\frac{e^{-i \kappa \ell \theta}}{\sqrt{\mathcal{N}_{\ell}}}\left[\begin{array}{c}
0 \\
\tilde{r}^{\ell}
\end{array}\right]
$$

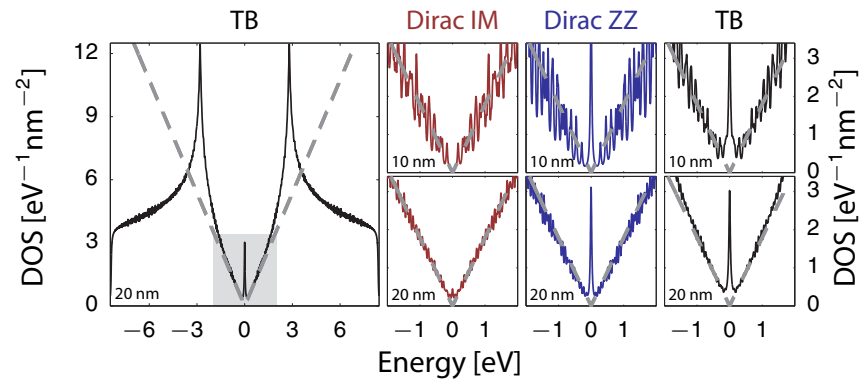

FIG. 2. (Color online) DOS for graphene nanodisk in Dirac ZZ and IM, and TB treatments, broadened by a loss of $\hbar \eta=24 \mathrm{meV}$, with disk diameter indicated. The asymptotic low-energy, bulk graphene DOS is indicated in dashed gray. The region of approximately linear DOS is indicated by gray shading.

with normalization $\mathcal{N}_{\ell}$ (see SM). The phenomenologically introduced cutoff angular quantum number $\ell_{\max }$ is required to avoid a divergence of the density of states at zero energy, and is chosen to ensure a total number of zero-energy states (including spin and valley degeneracy) $\sim 2 \pi R / 3 a_{\mathrm{LC}}[23]$ (see $\mathrm{SM})$. Except for the $\ell=0$ case, the zero-energy states are predominately localized at the disk edge, with a characteristic localization length $R\left[1-e^{-1 / 2(\ell+1)}\right]$ (see SM).

In Fig. 2 we show the resulting noninteracting density of states (DOS), phenomenologically broadened by an electron collision rate $\eta$, computed as $\operatorname{DOS}(\epsilon)=\frac{2}{\pi \mathcal{A}} \sum_{\nu} \operatorname{Im}\left[\left(\epsilon_{\nu}-\epsilon-\right.\right.$ $i \hbar \eta)^{-1}$ ] with $\mathcal{A}$ denoting the sample area and with $\sum_{\nu}$ denoting summation over all states $v$ (excluding spin, which contributes a factor 2). Also, the DOS for a TB model is shown, for a bond-centered disk. A key feature of both TB and Dirac ZZ treatments is a prominent peak at zero energy associated with edge states, which is not reproduced in either Dirac IM or in bulk approximations. Additionally, due to breaking of valley and azimuthal symmetry in TB the interstate energy-level spacing is overestimated in Dirac treatments relative to TB. Nevertheless, the total number of edge and bulk states in Dirac ZZ and TB is in good agreement (see SM). Due to the absence of edge states in Dirac IM vis-à-vis its presence in TB, we focus in the following on Dirac ZZ. Finally, we note the complete absence of nonconical dispersion effects, e.g., trigonal warping [19] and van Hove quasisingularities at $\pm t_{A B}$ [29], in the Dirac treatment, whose exemption, however, is expected to be unimportant in low-energy plasmonics.

Random-phase approximation. To compute the optical response of graphene disks in both TB and Dirac approaches, the first step is to evaluate the noninteracting polarizability $[11,30]$ :

$$
\chi^{0}\left(\mathbf{r}, \mathbf{r}^{\prime} ; \omega\right)=2 \sum_{\nu v^{\prime}}\left(f_{v}-f_{v^{\prime}}\right) \frac{\boldsymbol{\psi}_{v^{\prime}}^{\dagger}(\mathbf{r}) \boldsymbol{\psi}_{v}(\mathbf{r}) \boldsymbol{\psi}_{\nu}^{\dagger}\left(\mathbf{r}^{\prime}\right) \boldsymbol{\psi}_{v^{\prime}}\left(\mathbf{r}^{\prime}\right)}{\epsilon_{v}-\epsilon_{v^{\prime}}-\hbar(\omega+i \eta)},
$$

where $f_{v}$ denotes Fermi-Dirac equilibrium functions evaluated at energy $\epsilon_{\nu}$, and electron relaxation is included phenomenologically through a finite rate $\eta$. We give explicit expressions for the Dirac-disk polarizability in the SM.

The random-phase approximation (RPA) is instated by coupling the induced charge density $\rho(\mathbf{r})$ to the total field via 
$\chi^{0}$, leading to a self-consistent integral equation, reading, in operator notation, as $\rho=e^{2} \chi^{0}\left(\boldsymbol{\phi}^{\text {ext }}+\mathbf{V} \rho\right)$, with $\mathbf{V}$ denoting the Coulomb interaction and $\boldsymbol{\phi}^{\text {ext }}$ an external potential [31]. Henceforth, depending on the choice for single-particle states used in constructing $\chi^{0}$, we distinguish between approaches by the self-evident notation RPA@Dirac and RPA@TB. In the SM we elucidate the technical details for efficiently computing RPA@Dirac via an angular momentum decomposition, and follow the scheme introduced in Ref. [11] for RPA@TB. The computational complexity of these approaches is discussed and compared in the SM. The optical absorption cross section, i.e., the absorbed power relative to the intensity of an incident plane wave, relates to the induced charge density via $\sim \omega \operatorname{Im}[p(\omega)]$, with $p(\omega)$ denoting the dipole moment obtained from $\rho(\mathbf{r})$.

Local response. For comparison with the two quantum approaches described above, we also consider the traditional, classical approach, wherein the induced charge density in graphene is determined from the well-known bulk localresponse (LR) conductivity with intra- and interband terms $\sigma_{\mathrm{B}}(\omega)=\sigma_{\text {intra }}(\omega)+\sigma_{\text {inter }}(\omega)$ [32]. The interband term induces a redshift [33] of the dipolar plasmon resonance with decreasing radius, but not to the extent observed in TB-RPA calculations [11]. For the electrostatic disk, the LR problem is solved most elegantly by using a polynomial expansion technique, as explicated by Fetter [34], and summarized for completeness in the SM, allowing a semianalytical solution requiring only a numerical matrix inversion. Applying this technique, we find that the singly radially quantized dipole plasmon resonance, $\omega_{\mathrm{dp}}$, being the resonance of primary relevance in nanoscopic disks, relates to the total LR conductivity $\sigma(\omega)$ via $\omega_{\mathrm{dp}} / \sigma\left(\omega_{\mathrm{dp}}\right)=\zeta / 2 i \varepsilon_{0} \varepsilon_{\mathrm{B}} R$, with $\varepsilon_{\mathrm{B}}$ denoting the background dielectric constant and $\zeta \approx 1.0977$ accounting for the disk geometry [35]. An intraband approximation then entails the scaling $\omega_{\mathrm{dp}} \propto 1 / R^{1 / 2}$.

Although the bulk LR conductivity $\sigma_{\mathrm{B}}(\omega)$ is usually derived from a starting point of a continuum of bulk graphene Dirac states, it may as well be derived from the large-radius limit of the finite sample's conductivity using the states $\boldsymbol{\psi}_{l n}^{\kappa}$ from Eq. (3). Specifically, in the LR limit, the current response due to an $x$-polarized field is obtained from the conductivity [31]:

$$
\sigma(\omega)=\frac{2 i e^{2} \omega}{\mathcal{A}} \sum_{\nu v^{\prime}}\left(f_{v}-f_{v^{\prime}}\right) \frac{\left|\left\langle\boldsymbol{\psi}_{v}|x| \boldsymbol{\psi}_{v^{\prime}}\right\rangle\right|^{2}}{\epsilon_{v}-\epsilon_{v^{\prime}}-\hbar(\omega+i \eta)} .
$$

Considering the Dirac ZZ states in Eqs. (3) and (4) this gives rise to two distinct terms, one tending asymptotically to $\sigma_{\mathrm{B}}(\omega)$ with increasing radius, originating from bulk-to-bulk transitions $\left|\left\langle\boldsymbol{\psi}_{l n}^{\kappa}|x| \boldsymbol{\psi}_{l^{\prime} n^{\prime}}^{\kappa}\right\rangle\right|^{2}$, and one novel term originating from edge-to-bulk transitions:

$$
\sigma_{\mathrm{E}}(\omega)=\frac{4 i e^{2} \omega}{\mathcal{A}} \sum_{\kappa \ln \ell}\left(f_{l n}-f_{0}\right) \frac{\left|\left\langle\boldsymbol{\psi}_{l n}^{\kappa}|x| \boldsymbol{\phi}_{\ell}^{\kappa}\right\rangle\right|^{2}}{\epsilon_{l n}^{2}-\hbar^{2}(\omega+i \eta)^{2}},
$$

with $f_{0}$ denoting the Fermi-Dirac function at zero energy, and $\epsilon_{l n}$ denoting the Dirac ZZ energies corresponding to $\boldsymbol{\psi}_{l n}^{\kappa}$. This edge contribution, physically representing all interactions between occupied zero-energy edge states and unoccupied nonzero-energy bulk states, can be worked out explicitly as

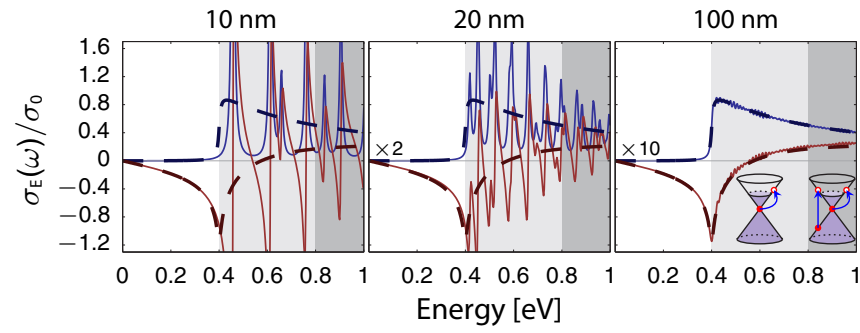

FIG. 3. (Color online) Edge-state conductivity in graphene nanodisks (doped to $0.4 \mathrm{eV}$ ) normalized to $\sigma_{0}=e^{2} / 4 \hbar$ calculated with finite damping $\hbar \eta=6 \mathrm{meV}$. Disk diameter indicated in titles; note the scaling factor in the center and right-hand graphs. Explicit evaluation of Eq. (8) given in full, and large-radius limit, Eq. (9), in bold dashed; real and imaginary parts in blue and red, respectively. The region of edge-to-bulk transitions is indicated in gray, while the region of concurrent edge-to-bulk and interband transitions is dark-gray, and illustrated schematically.

a summation over the Bessel function zeros $\beta_{l n}$ (see SM for details). In the low-temperature limit, assuming positive $\epsilon_{\mathrm{F}}$, the edge-state conductivity becomes

$$
\sigma_{\mathrm{E}}(\omega)=\frac{-16 i e^{2}}{\pi \hbar} \frac{\omega}{\omega_{R}} \sum_{\ell=0}^{\ell_{\max }} \sum_{n}^{\hbar \omega_{R} \beta_{\ell n}>\epsilon_{\mathrm{F}}} \frac{\ell+1}{\beta_{\ell n}^{5}\left[1-\left(\frac{\omega+i \eta}{\beta_{\ell n} \omega_{R}}\right)^{2}\right]} .
$$

Remarkably, the above expression allows a simple asymptotic form in the large-radius limit $R \rightarrow \infty$. Replacing the angular momenta $\ell+1$ by their average at fixed energy $\ell+1 \rightarrow\langle\ell+$ $1\rangle_{\epsilon} \simeq \xi \epsilon / \hbar \omega_{R}$, with proportionality constant $\xi=4 / 3 \pi$ (see SM), introducing the bulk-energy substitution $\beta_{\ell n} \rightarrow \epsilon / \hbar \omega_{R}$, and transforming the $\ell n$ summations into integrals over $\epsilon_{\mathrm{F}} \leqslant$ $\epsilon<\infty$, we find

$$
\sigma_{\mathrm{E}}^{\infty}(\omega)=\xi \frac{2 e^{2}}{\pi \hbar} \frac{v_{\mathrm{F}}}{\omega R}\left[i \ln \left|\frac{\epsilon_{\mathrm{F}}^{2}-\hbar^{2} \omega^{2}}{\epsilon_{\mathrm{F}}^{2}}\right|+\pi \theta\left(\hbar \omega-\epsilon_{\mathrm{F}}\right)\right],
$$

shown here, for simplicity, in the low-loss limit $\eta \rightarrow 0^{+}$[36]. Interestingly, Eq. (9) shows that the inclusion of edge states opens a dispersive channel scaling with $\omega_{R}=v_{\mathrm{F}} / R$, mathematically reminiscent of, but physically distinct from, the scaling phenomenologically introduced in Kreibig damping [37] and recently derived from the viewpoint of nonlocal diffusion dynamics [38]. In addition to Landau damping due to vertical transitions, as included in $\sigma_{\text {inter }}(\omega)$ for $\hbar \omega \geqslant 2 \epsilon_{\mathrm{F}}$, edgeto-bulk transitions allow nonvertical transitions at $\hbar \omega \geqslant \epsilon_{\mathrm{F}}$, with the necessary momentum supplied by the structural truncation with a strength proportional to $1 / R$. In Fig. 3 we consider $\sigma_{\mathrm{E}}(\omega)$ and compare with $\sigma_{\mathrm{E}}^{\infty}(\omega)$ for three disk diameters. At smaller diameters $\sigma_{\mathrm{E}}(\omega)$ and $\sigma_{\mathrm{E}}^{\infty}(\omega)$ differ substantially in the region $\hbar \omega>\epsilon_{\mathrm{F}}$ with $\sigma_{\mathrm{E}}$ exhibiting peaks at discrete transitional energies $\hbar \omega \simeq \epsilon_{\ell n}$; as the diameter is increased, and the energy difference between distinct transitional energies decreases accordingly, $\sigma_{\mathrm{E}}$ approaches $\sigma_{\mathrm{E}}^{\infty}$ asymptotically, as anticipated. We note that a generally good agreement is apparent, even for small disks, when $\hbar \omega<\epsilon_{\mathrm{F}}$. The importance of the edge-state conductivity vis-á-vis the bulk conductivity diminishes with increasing diameter due to the $1 / R$ scaling of $\sigma_{\mathrm{E}}(\omega)$. Nonetheless, even at large disk diameters, e.g., $20 \mathrm{~nm}$, the maximal edge-state conductivity is 
still on the order of $\sim 0.4 \sigma_{0}$, while the magnitude of vertical interband transitions roughly amounts to $\sigma_{0}$.

Hydrodynamic response. The noninteracting polarizability, the key constituent of the RPA, as considered in Eq. (5), accounts not only for the discretized and individual nature of the allowable states, but also for the nonlocal nature of the electromagnetic response, manifest in the finitude of $\chi^{0}\left(\mathbf{r}, \mathbf{r}^{\prime} ; \omega\right)$ for $\mathbf{r} \neq \mathbf{r}^{\prime}$. An approximate accounting of nonlocal response can be facilitated by a hydrodynamic model [33]:

$$
\left(1+\frac{\beta^{2}}{\omega^{2}} \nabla_{\|}^{2}\right) \rho(\mathbf{r})=\frac{i \sigma(\omega)}{\omega} \nabla_{\|}^{2} \phi(\mathbf{r})
$$

with $\nabla_{\|}^{2}$ being the two-dimensional Laplacian, and with plasma velocity denoted by $\beta^{2}=\frac{3}{4} v_{\mathrm{F}}^{2}$ (see $\mathrm{SM}$ ). For brevity, we will denote hydrodynamic calculations with a backbone conductivity $\sigma(\omega)$ by $[\sigma]^{\mathrm{H}}(\omega)$. The primary effect of the hydrodynamic model is to introduce a blueshift, which, in $\left[\sigma_{\mathrm{B}}\right]^{\mathrm{H}}(\omega)$, approximately amounts to a shift $\delta \omega_{\mathrm{dp}} \simeq 1.27 \omega_{R}^{2} / \omega_{\mathrm{dp}}$. Predictions of the hydrodynamic model at the level $\left[\sigma_{\mathrm{B}}\right]^{\mathrm{H}}(\omega)$ agree excellently with predictions of RPA@Dirac IM as we show in the SM. This underscores the accuracy of a hydrodynamic description, since RPA@Dirac IM neglects the existence of edge states, and thus, at large radii, is modified primarily by nonlocal effects. Moreover, through this, we qualitatively explain the blueshift predicted by RPA@TB in armchair nanostructures $[39,40]$ as a hydrodynamic shift.

Results and discussion. Figure 4 depicts the absorption cross-sectional efficiency, i.e., cross section normalized to disk area, for a normally incident excitation field, i.e., propagating along $z$, of graphene nanodisks for different diameters, contrasting results obtained by LR with and without hydrodynamic and edge-state conductivity, RPA@TB, and RPA@Dirac ZZ. A fundamental feature of RPA@TB, not captured by any of the continuum models, is a polarization dependence, considered in Fig. 4 for $x$ - and $y$-polarized incident fields, of the optical response due to the discrete nature of the description. For smaller disks, only few EHPs contribute, leading to a strong polarization dependence. For larger disks, as the number of contributing EHPs increases, and the collective nature of the plasmon emerges, this dependence diminishes rapidly.

The primary feature of both RPA@TB and RPA@Dirac $\mathrm{ZZ}$ for disk diameters larger than approximately $14 \mathrm{~nm}$, is the emergence of a broad dominant plasmonic resonance redshifted with respect to the LR bulk predictions. Comparison with $\left[\sigma_{\mathrm{B}}+\sigma_{\mathrm{E}}\right]^{\mathrm{H}}(\omega)$ and $\left[\sigma_{\mathrm{B}}+\sigma_{\mathrm{E}}^{\infty}\right]^{\mathrm{H}}(\omega)$ agrees qualitatively. A similar redshift is reproduced, $\underset{\sim}{\sim} \omega_{R}^{2} / \omega_{\mathrm{dp}} \propto 1 / R^{3 / 2}$ (see $\mathrm{SM}$ ), but slightly underestimated in magnitude due to the assumption of a constant total field, inherent to the dipole approximation in Eq. (6), contrasting the actual electric field distribution of the plasmon, which is significantly concentrated near the edge [41]. Furthermore, the dipole resonance in RPA@TB is damped and broadened to a larger degree than both RPA@Dirac ZZ and $\left[\sigma_{\mathrm{B}}+\sigma_{\mathrm{E}}\right]^{\mathrm{H}}(\omega)$ as a result of the explicit breaking of azimuthal and valley symmetry in the discrete treatment, permitting additional dipole-allowed transitions.

In conclusion, the redshift observed between predictions of RPA@TB and bulk LR calculations arises from the competing

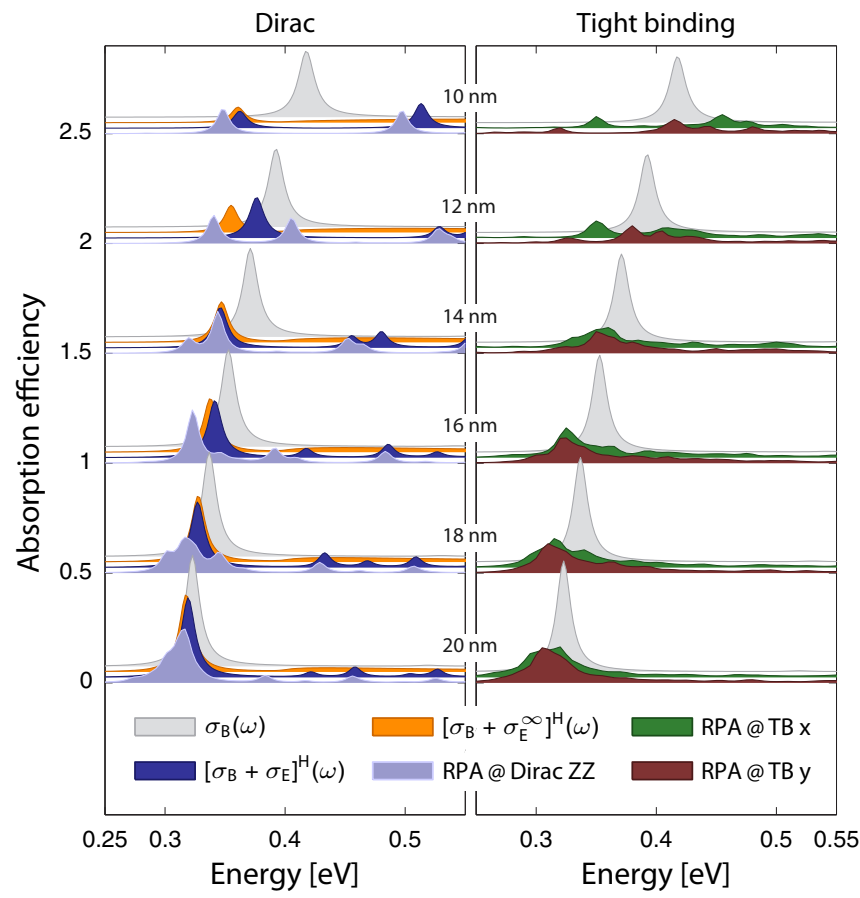

FIG. 4. (Color online) Absorption cross-sectional efficiency at normal incidence of graphene nanodisks calculated via LR, hydrodynamics with bulk and edge-state conductivities, RPA@Dirac $\mathrm{ZZ}$, and RPA@TB (for $x$ - and $y$-polarized light) for disks of increasing diameter. The sample is considered doped to $\epsilon_{\mathrm{F}}=0.4 \mathrm{eV}$, with electron relaxation-rate $\hbar \eta=6 \mathrm{meV}$, and at a temperature $T=300 \mathrm{~K}$. Spectra for different diameters are offset by 0.5 , while individual spectra at identical diameters are offset by 0.025 . Spectra for intermediate diameters available in SM.

effects of edge conductivity and nonlocal response, with the former prevailing, shifting the dipole resonance $\omega_{\mathrm{dp}}$ to the red and blue, respectively, with a strength $\underset{\sim}{\propto} \omega_{R}^{2} / \omega_{\mathrm{dp}}$ in both cases. The simultaneous accounting of both effects is thus of paramount importance in semiclassically reproducing the key plasmonic features of full RPA@TB predictions, with significant corrections even at relatively large diameters $\sim 20 \mathrm{~nm}$. Our identification and effective description of this additional dispersive channel via edge states illustrates an important difference between graphene and metal plasmonics. Additionally, the equivalent size-dependent scaling with, e.g., nonlocal corrections, accentuates its high-ranking position in the hierarchy of nonclassical corrections for plasmonics at the nanoscale. Encouragingly, the salient features of Eq. (9) are geometry independent, and we accordingly predict that the simple analytical term can be qualitatively extended to other graphene nanostructures, e.g., by substituting $R \rightarrow 2 A / C$ with $A$ and $C$ denoting system area and circumference, respectively, reasonable for sufficiently smooth boundaries. Finally, generalizations to a much wider class of systems supporting edge or surface states appear feasible, e.g., in topological insulators such as bismuth bilayers [42] or silicene [43], $\mathrm{MoS}_{2}$ nanotriangles [44], nanostructures with $\mathrm{Ag}(111)$ facets [45], or indeed in any finite bipartite system which generally supports zero-energy localized states [46]. 
Acknowledgments. We thank Wei Yan for many fruitful discussions, helpful suggestions, and continued interest. The Center for Nanostructured Graphene is sponsored by the
Danish National Research Foundation, Project DNRF58. This work was also supported by the Danish Council for Independent Research, Project 1323-00087.
[1] M. S. Tame, K. R. McEnery, Ş. K. Özdemir, J. Lee, S. A. Maier, and M. S. Kim, Nat. Phys. 9, 329 (2013).

[2] K. Ostrikov, U. Cvelbar, and B. Murphy, J. Phys. D: Appl. Phys. 44, 174001 (2011).

[3] J. Henzie, J. Lee, M.H. Lee, W. Hasan, and T. W. Odom, Annu. Rev. Phys. Chem. 60, 147 (2009).

[4] A. N. Grigorenko, M. Polini, and K. S. Novoselov, Nat. Photonics 6, 749 (2012).

[5] Y. V. Bludov, A. Ferreira, N. M. R. Peres, and M. I. Vasilevskiy, Int. J. Mod. Phys. B 27, 1341001 (2013).

[6] F. J. García de Abajo, ACS Photonics 1, 135 (2014).

[7] M. Jablan, H. Buljan, and M. Soljačić, Phys. Rev. B 80, 245435 (2009).

[8] F. H. L. Koppens, D. E. Chang, and F. J. García de Abajo, Nano Lett. 11, 3370 (2011)

[9] V. W. Brar, M. S. Jang, M. Sherrott, J. J. Lopez, and H. A. Atwater, Nano Lett. 13, 2541 (2013).

[10] H. Yan, T. Low, W. Zhu, Y. Wu, M. Freitag, X. Li, F. Guinea, P. Avouris, and F. Xia, Nat. Photonics 7, 394 (2013).

[11] S. Thongrattanasiri, A. Manjavacas, and F. J. García de Abajo, ACS Nano 6, 1766 (2012).

[12] S. Thongrattanasiri, A. Manjavacas, P. Nordlander, and F. J. García de Abajo, Laser Photon. Rev. 7, 297 (2013).

[13] A. Manjavacas, F. Marchesin, S. Thongrattanasiri, P. Koval, P. Nordlander, D. Sánchez-Portal, and F. J. García de Abajo, ACS Nano 7, 3635 (2013).

[14] Z. Fang, S. Thongrattanasiri, A. Schlather, Z. Liu, L. Ma, Y. Wang, P. M. Ajayan, P. Nordlander, N. J. Halas, and F. J. García de Abajo, ACS Nano 7, 2388 (2013).

[15] X. Zhu, W. Wang, W. Yan, M. B. Larsen, P. Bøggild, T. G. Pedersen, S. Xiao, J. Zi, and N. A. Mortensen, Nano Lett. 14, 2907 (2014).

[16] K. Kim, S. Coh, C. Kisielowski, M. F. Crommie, S. G. Louie, M. L. Cohen, and A. Zettl, Nat. Commun. 4, 2723 (2013).

[17] S. K. Hämäläinen, Z. Sun, M. P. Boneschanscher, A. Uppstu, M. Ijäs, A. Harju, D. Vanmaekelbergh, and P. Liljeroth, Phys. Rev. Lett. 107, 236803 (2011).

[18] X. Jia, M. Hofmann, V. Meunier, B. G. Sumpter, J. CamposDelgado, J. M. Romo-Herrera, H. Son, Y.-P. Hsieh, A. Reina, J. Kong, M. Terrones, and M. S. Dresselhaus, Science 323, 1701 (2009).

[19] A. H. Castro Neto, F. Guinea, N. M. R. Peres, K. S. Novoselov, and A. K. Geim, Rev. Mod. Phys. 81, 109 (2009).

[20] In the present study we forfeit consideration of armchair lattice termination, which induces valley admixing [21], and thus examine the two-spinor Dirac equations with $\hat{\mathcal{H}}_{\mathrm{D}}^{+}=v_{\mathrm{F}} \boldsymbol{\sigma} \cdot \hat{\boldsymbol{p}}$ and $\hat{\mathcal{H}}_{\mathrm{D}}^{-}=v_{\mathrm{F}} \boldsymbol{\sigma}^{*} \cdot \hat{\boldsymbol{p}}$.

[21] L. Brey and H. A. Fertig, Phys. Rev. B 73, 195408 (2006).

[22] E. McCann and V. I. Fal'ko, J. Phys.: Condens. Matter 16, 2371 (2004).

[23] A. R. Akhmerov and C. W. J. Beenakker, Phys. Rev. B 77, 085423 (2008).
[24] M. V. Berry and R. J. Mondragon, Proc. R. Soc. London, Ser. A 412, 53 (1987)

[25] N. M. R. Peres, J. N. B. Rodrigues, T. Stauber, and J. M. B. Lopes dos Santos, J. Phys.: Condens. Matter 21, 344202 (2009).

[26] B. Wunsch, T. Stauber, and F. Guinea, Phys. Rev. B 77, 035316 (2008).

[27] M. Grujić, M. Zarenia, A. Chaves, M. Tadić, G. A. Farias, and F. M. Peeters, Phys. Rev. B 84, 205441 (2011).

[28] See Supplemental Material at http://link.aps.org/supplemental/ 10.1103/PhysRevB.90.241414 for additional details, including derivation of results, supporting simulations, and description of methodology.

[29] A. Zhou and W. Sheng, J. Appl. Phys. 112, 094313 (2012).

[30] A. Manjavacas and F. J. García de Abajo, Nat. Commun. 5, 3548 (2014).

[31] G. Grosso and G. P. Parravicini, Solid State Physics, 2nd ed. (Academic, New York, 2014).

[32] L. A. Falkovsky and A. A. Varlamov, Eur. Phys. J. B 56, 281 (2007).

[33] W. Wang and J. M. Kinaret, Phys. Rev. B 87, 195424 (2013).

[34] A. L. Fetter, Phys. Rev. B 33, 5221 (1986).

[35] Similar results were found in Ref. [14], albeit with the less precise value of $\zeta \approx 0.995$ likely due to use of a general, but less tailored, numerical approach. Precise evaluation of $\zeta$ is crucial to accurately assess the actual resonance shifts of RPA@TBA relative to LR bulk calculations.

[36] Finite loss, $\eta>0$, is included through the rigorous substitutions $\omega_{R} / \omega \rightarrow \omega_{R} \omega /(\omega+i \eta), \pi \theta\left(\omega-\tilde{\epsilon}_{\mathrm{F}}\right) \rightarrow \arctan \left[\left(\tilde{\epsilon}_{\mathrm{F}}+\omega\right) / \eta\right]-$ $\arctan \left[\left(\tilde{\epsilon}_{\mathrm{F}}-\omega\right) / \eta\right]$, and $\ln \left|\left(\tilde{\epsilon}_{\mathrm{F}}^{2}-\omega^{2}\right) / \tilde{\epsilon}_{\mathrm{F}}^{2}\right| \rightarrow \frac{1}{2} \ln \left\{\left[\left(\tilde{\epsilon}_{\mathrm{F}}-\omega\right)^{2}+\right.\right.$ $\left.\left.\eta^{2}\right] / \tilde{\epsilon}_{\mathrm{F}}^{2}\right\}+\frac{1}{2} \ln \left\{\left[\left(\tilde{\epsilon}_{\mathrm{F}}+\omega\right)^{2}+\eta^{2}\right] / \tilde{\epsilon}_{\mathrm{F}}^{2}\right\}$, where $\tilde{\epsilon}_{\mathrm{F}}=\epsilon_{\mathrm{F}} / \hbar$.

[37] U. Kreibig and C. Fragstein, Z. Phys. 224, 307 (1969).

[38] N. A. Mortensen, S. Raza, M. Wubs, T. Søndergaard, and S. I. Bozhevolnyi, Nat. Commun. 5, 3809 (2014).

[39] S. Thongrattanasiri and F. J. García de Abajo, Phys. Rev. Lett. 110, 187401 (2013).

[40] W. Wang, T. Christensen, A.-P. Jauho, K. S. Thygesen, M. Wubs, and N. A. Mortensen, arXiv:1410.0537.

[41] W. Wang, P. Apell, and J. Kinaret, Phys. Rev. B 84, 085423 (2011).

[42] I. K. Drozdov, A. Alexandradinata, S. Jeon, S. Nadj-Perge, H. Ji, R. J. Cava, B. Andrei Bernevig, and A. Yazdani, Nat. Phys. 10, 664 (2014).

[43] K. Kikutake, M. Ezawa, and N. Nagaosa, Phys. Rev. B 88, 115432 (2013).

[44] M. V. Bollinger, K. W. Jacobsen, and J. K. Nørskov, Phys. Rev. B 67, 085410 (2003).

[45] T. C. Hsieh, T. Miller, and T.-C. Chiang, Phys. Rev. Lett. 55, 2483 (1985).

[46] M. Inui, S. A. Trugman, and E. Abrahams, Phys. Rev. B. 49, 3190 (1994). 